\title{
Construction of Soluble Mamu-B*1703, a Class I Major Histocompatibility Complex of Chinese Rhesus Macaques, Monomer and Tetramer Loaded with a Simian Immunodeficiency Virus Peptide
}

\author{
Dongyun Ouyang ${ }^{1}$, Xiaoying Wang ${ }^{1}$, Xianhui He ${ }^{1,3}$, Lihui Xu ${ }^{2}$, Huanjing Shi ${ }^{1}$, Qi Gao ${ }^{1}$ and He Guo ${ }^{1}$
}

Chinese-descent rhesus macaques have become more prevalent for HIV infection and vaccine investigation than Indian-origin macaques. Most of the currently available data and reagents such as major histocompatibility complex (MHC) class I tetramers, however, were derived from Indian-origin macaques due to the dominant use of these animals in history. Although there are significant differences in the immunogenetic background between the two macaque populations, they share a few of common MHC class I alleles. We reported in this study the procedure for preparation of a soluble Mamu-B*1703 (a MHC class I molecule of Chinese macaques) monomer and tetramer loaded with a dominant simian immunodeficiency virus (SIV) epitope IW9 (IRYPKTFGW) that was identified to be Mamu- $B * 1701$-restricted in Indian macaques. The DNA fragment encoding the Mamu-B*1703 extracellular domain fused with a BirA substrate peptide (BSP) was amplified from a previously cloned cDNA and inserted into a prokaratic expression vector. In the presence of the antigenic peptide IW9 and light chain $\beta_{2}$-microglobulin, the expressed heavy chain was refolded into a soluble monomer. After biotinylation, four monomers were polymerized as a tetramer by phycoerythrin-conjugated streptavidin. The tetramer, having been confirmed to have the right conformation, was a potential tool for investigation of antigen-specific $\mathrm{CDB}^{+}$ T-lymphocytes in SIV vaccine models of Chinese macaques. And our results also suggested that some antigenic peptides reported in Indian-origin macaques could be directly recruited as ligands for construction of Chinese macaque MHC tetramers. Cellular \& Molecular Immunology. 2009;6(2):117-122.

Key Words: Mamu-B*17, MHC class I, rhesus macaque, tetramer

\section{Introduction}

Simian immunodeficiency virus (SIV)-infected rhesus macaques (Macaca mulatta) are served as human immunodeficiency virus (HIV)-infection and vaccine models (1). But different populations with variant backgrounds of major histocompatibility complexes (MHC) have different susceptibilities and responses to SIV infection, as have been observed in Chinese- and Indian-origin rhesus macaques

\footnotetext{
${ }^{1}$ Institute of Tissue Transplantation and Immunology, College of Life Science and Technology, Jinan University, Guangzhou 510632, China;

${ }^{2}$ Institute of Bioengineering, College of Life Science and Technology, Jinan University, Guangzhou 510632, China;

${ }^{3}$ Correspondence to: Dr. Xianhui He, Institute of Tissue Transplantation and Immunology, College of Life Science and Technology, Jinan University, Guangzhou 510632, China. Tel: +86-20-8522-0679, Fax: +86-20-85221337, E-mail: thexh@jnu.edu.cn

Received Jan 8, 2008. Accepted Feb 16, 2009.

C2009 Chinese Society of Immunology and University of Science \& Technology of China
}

(2-5). Recently, Chinese-descent rhesus macaques have become more prevalent for HIV infection and vaccine investigation than Indian-origin macaques. Most of the currently available data and reagents such as MHC class I tetramers, however, are derived from Indian-origin macaques due to the dominant use of these animals in history. Thus, the critical reagents such as MHC class I tetramers are urgently required for vaccine researches in Chinese macaques.

MHC class I alleles play a key role in cellular immune responses to intracellular pathogens. Peptides processed from the pathogens are carried by these MHC molecules to the cell surface and presented to $\mathrm{CD} 8^{+}$cytotoxic T-lymphocytes (CTLs) by which the infected cells are eventually destructed. There are several methods to evaluate the antigen-specific $\mathrm{CD}^{+}$CTLs, including intracellular cytokine staining, ELISpot-cytokine assay, chromium release assay and MHC class I tetramer staining (6). Owing to the complicated performance and thus increasing cell apoptosis, intracellular cytokine staining and secreted cytokine staining (ELISpot) are not ideal methods. Chromium release assay is also not popularly welcome due to its dangerous irradiation. In contrast, MHC class I tetramer staining is the only method that can directly enumerate the frequency of antigen-specific 
$\mathrm{CD}^{+}$CTLs in spite of the laborious procedure for preparation of tetramers.

Previously, we have established a simplified procedure for preparing HLA-A*0201 tetramer loaded with human cytomegalovirus pp65 $495-503$ peptide (NLVPMVATV, NLV) or influenza virus matrix protein $\mathrm{MP}_{58-66}$ peptide (GILGFVFTL, GIL) (7) and these tetramers have been used to analyze the details of antigen-specific CTLs in Chinese populations $(8,9)$. In a tetramer, four monomers are linked together by phycoerythrin (PE)-conjugated streptavidin. Each monomer, which embodies the conformation of HLA-A*0201 presenting antigenic peptide on cell surfaces, is composed of the extracellular domain of the heavy chain, the light chain $\beta_{2}$-microglobulin $\left(\beta_{2} \mathrm{~m}\right)$ and the antigenic peptide. By polymerization of the monomers, the tetramers' avidity for cognate $\mathrm{CD}^{+}$CTLs is increased greatly, thus allowing direct staining of antigen-specific CTLs. Previously we have identified a novel MHC class I allele of Chinese-descent rhesus macaques, Матu- $B^{*} 1703$ (10), which is highly homologous with Mamu-B*1701 of Indian-origin macaques. Similar procedure with necessary changes was adopted in this study for construction of Mamu-B*1703 tetramer loaded with a dominant epitope IW9 corresponding to residues 165-173 (IRYPKTFGW) of SIVnef protein (11, 12). Its conformation was confirmed by monoclonal antibody (W6/32) recognition assay. These results provided an important basis for further investigation of antigen-specific $\mathrm{CD}^{+}$CTLs in SIV animal models of Chinese macaques.

\section{Materials and Methods}

\section{Reagents}

Plasmid pET-3d was purchased from Novagen (Madison, WI, USA). Nco I, BamH I, T 4 DNA ligase, protein molecular weight (MW) markers and high fidelity PrimeSTAR HS DNA polymerase were purchased from TaKaRa (Dalian, China). MonoQ 5/50 GL column was obtained from Amersham (Uppsala, Sweden). R-phycoerythrin-conjugated streptavidin (PE-streptavidin) was obtained from Molecular Probes (Eugene, OR, USA). The biotinylation enzyme, BirA, was purchased from Avidity (Denver, CO, USA). Isopropyl$\beta$-D-thiogalactoside (IPTG), diaminobenzidine (DAB) and all the other chemicals used were of analytical reagent grade. Mouse-anti-human HLA-A*0201 serum was raised by our laboratory (13). PE-conjugated mouse-anti-human HLAABC (clone W6/32) was purchased from eBioscience (San Diego, CA, USA).

\section{Bacterial strains}

Escherichia coli (E. coli) strain DH5 $\alpha$ and BL21 (DE3) were obtained from Novagen (Madison, WI, USA).

\section{Peptide synthesis}

The antigenic peptide IW9 was synthesized by Invitrogen Biotechnology Co. (Shanghai, China) and purified to purity of $>95 \%$. The peptide was dissolved in dimethyl sulfoxide (DMSO) at a final concentration of $10 \mathrm{mg} / \mathrm{ml}$ and aliquots were stored at $-70^{\circ} \mathrm{C}$.

\section{Construction of the expression vector}

Mamu- $B^{*} 1703$ sequence cloned in pMD19-T vector previously reported by us (10) was used as a initial template for amplification of the fragment encoding the extracellular domain of Mamu-B*1703 heavy chain fused with a BirA substrate peptide (LHHILDAQKMVWNHR) through a linker Gly-Ser. A three-round PCR was performed with an initial denaturation at $94^{\circ} \mathrm{C}$ for 2 min followed by 35 cycles of $94^{\circ} \mathrm{C}$ for $30 \mathrm{~s}, 58^{\circ} \mathrm{C}$ for $30 \mathrm{~s}$ and $72^{\circ} \mathrm{C}$ for $60 \mathrm{~s}$, and a final extension at $72^{\circ} \mathrm{C}$ for $10 \mathrm{~min}$. In the first-round PCR, the primers for reaction tube A were: Forward-1703 (5'-TAT ACC ATG GGT TCT CAT TCT ATG AAA TAT TTT TAT ACC TCC GTG TCC CGG CCC GG-3') and BackmutBamH (5'-GTC TTT GGG GGA TCT GCG CGC TGC AGC GTC T-3'), and the primers for reaction tube B were Forword-mutBamH (5'-AGC GCG CAG ATC CCC CAA AGA C-3') and Backward-1703 (5'-CTG TGC ATC CAG AAT ATG ATG CAG AGA GCC CCA TCT TAG GGT GAG GGG CTC-3'). The BamH I restriction site was synonymously mutated. The second-round PCR was performed using cleaned tube $\mathrm{A}$ and tube $\mathrm{B}$ product mixture as template and using primers Forward-1703 and Backward1703. Finally, the second-round PCR products were extended using the primers Forward-1703 and Backward-2 (5'-AAT AGG ATC CTT AAC GAT GAT TCC ACA CCA TTT TCT GTG CAT CCA GAA TAT GAT GCA G-3'). By the three-round PCR, the final products were digested by Noc I and $B a m \mathrm{H}$ I and were inserted into plasmid pET-3d. Clones with a correct sized insert were identified by direct DNA sequencing (Invitrogen, Guangzhou), and such a target vector was named as $\mathrm{pET}-3 \mathrm{~d} /$ Mamu-B*1703-BSP.

\section{Expression and purification of Mamu-B*1703-BSP fusion protein}

The expression vector $\mathrm{pET}-3 \mathrm{~d} /$ Mamu- $^{*}$ 1703-BSP was transformed into $E$. coli BL21 (DE3) and the recombinant protein expression was induced with IPTG. Inclusion bodies of Mamu-B*1703-BSP fusion protein and human $\beta_{2}$ m were purified according to a previously described procedure (7, 14). Purified inclusion bodies were dissolved in $20 \mathrm{mmol} / \mathrm{L}$ 2-(N-morpholino) ethanesulfonic acid ( $\mathrm{pH}$ 6.0, containing 8 $\mathrm{mol} / \mathrm{L}$ urea, $10 \mathrm{mmol} / \mathrm{L}$ EDTA and $0.1 \mathrm{mmol} / \mathrm{L}$ DTT), respectively. The protein concentration was determined by measuring absorbance at $280 \mathrm{~nm}$ and $260 \mathrm{~nm}$, and calculated according to the empirical formula $\left(1.45 \times \mathrm{A}_{280}-0.74 \times \mathrm{A}_{260}\right.$ $=$ protein concentration in $\mathrm{mg} / \mathrm{ml}$ ). The protein was then aliquoted and stored at $-70^{\circ} \mathrm{C}$.

\section{SDS-PAGE and Western blotting}

Discontinuous SDS-PAGE was performed according to Laemmli (15), using a 15\% polyacryamide separating gel and a 5\% stacking gel. In brief, samples were subjected to SDS-PAGE for $45 \mathrm{~min}$ at $200 \mathrm{~V}$ and then the gel was stained by Coomassie Brilliant Blue R250. Gel images were taken by FluorChem SP imaging system (Alpha Innotech, San Leandro) and analyzed with AlphaEaseFC sofeware (Alpha 
Innotech). Western blotting was performed as described previously (13). Anti-HLA-A*0201 antiserum raised in mice was used as primary antibody.

\section{Construction of Mamu-B*1703-IW9 monomer and tetramer} Construction of Mamu-B*1703-IW9 monomer and tetramer was performed following the procedure described previously (7). In brief, Mamu-B*1703-BSP, $\beta_{2} \mathrm{~m}$ and IW9 peptide were refolded in the stirring refolding buffer $[0.1 \mathrm{~mol} / \mathrm{L}$ Tris- $\mathrm{HCl}$, $\mathrm{pH} 8.0$, containing $0.4 \mathrm{~mol} / \mathrm{L}$ L-arginine, $2 \mathrm{mmol} / \mathrm{L}$ EDTA, 5 $\mathrm{mmol} / \mathrm{L}$ reduced glutathione, $0.5 \mathrm{mmol} / \mathrm{L}$ oxidized glutathione, $0.2 \mathrm{mmol} / \mathrm{L}$ phenylmethyl sulfonyl fluoride (PMSF), prechilled to $10^{\circ} \mathrm{C}$ ]. Refolded Mamu-B*1703-IW9 monomer was biotinylated at the specific site on BSP by BirA according to the recommended procedure. Biotinylated monomer was then dialyzed against $10 \mathrm{mmol} / \mathrm{L}$ Tris- $\mathrm{HCl}$ buffer ( $\mathrm{pH}$ 8.0) and loaded onto MonoQ5/50 GL column preequilibrated with the same buffer. The column was eluted with a linear gradient of $0-150 \mathrm{mmol} / \mathrm{L} \mathrm{NaCl}$ using Akta UPC9000 system (Amersham, Uppsala, Sweden). The fractions (about $1 \mathrm{ml}$ ) were collected and determined by SDS-PAGE. The peak containing both Mamu-B*1703-BSP and $\beta_{2} \mathrm{~m}$ bands were collected and concentrated. The buffer was then changed to PBS (containing $0.2 \mathrm{mmol} / \mathrm{L}$ PMSF and $2 \mathrm{mmol} / \mathrm{L}$ EDTA) by ultrafiltration. The protein concentration was determined as described above and stored at $4^{\circ} \mathrm{C}$. Mamu-B*1703-IW9 tetramer was derived by mixing the biotinylated monomers with PE-streptavidin at a molar ratio of $4: 1$. The final tetrameric complex was stored at $4^{\circ} \mathrm{C}$.

\section{Identification of the Mamu-B*1703-IW9 tetramer}

Identification of the Mamu-B*1703-IW9 tetramer was performed as reported (16) with necessary changes. In brief, a 96-well plate was coated overnight at $4^{\circ} \mathrm{C}$ with $100 \mu$ of 5 $\mu \mathrm{g} / \mathrm{ml}$ monoclonal antibody ( $\mathrm{mAb}) \mathrm{W} 6 / 32$ in PBS. After blocked with blocking buffer $(5 \%$ non-fat milk in PBS-0.05\% Tween 20), $100 \mu \mathrm{l}$ of $2 \mu \mathrm{g} / \mathrm{ml} \beta_{2} \mathrm{~m}$, Mamu-

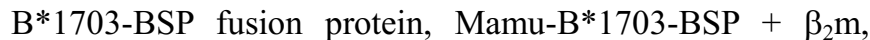
Mamu-B*1703/IW9 monomer or HLA-A*0201/NLV monomer (7) in blocking buffer were added in triplicates to the plate respectively and incubated at $37^{\circ} \mathrm{C}$ for $1 \mathrm{~h}$. The plate was then incubated with $100 \mu \mathrm{l}$ rabbit-anti- $\beta_{2} \mathrm{~m}$ antibody (Antibody Diagnostica Inc, USA) (1:800 in blocking buffer) and followed by incubation with HRP-conjugated goatanti-rabbit $\operatorname{IgG}(1: 4,000$ in blocking buffer). Finally, o-phenylenediamine (OPD) substrate was added into the wells and the absorbance at $490 \mathrm{~nm}$ was read on a Model 680 Microplate Reader (Bio-Rad).

\section{Results}

\section{Construction of Mamu-B*1703-BSP expression vector}

The DNA fragment encoding the ectodomain of MamuB*1703 heavy chain fused with BSP sequence by a Gly-Ser linker was constructed by the three-round PCR procedure as described in Materials and Methods. As there is also a $B a m \mathrm{H}$
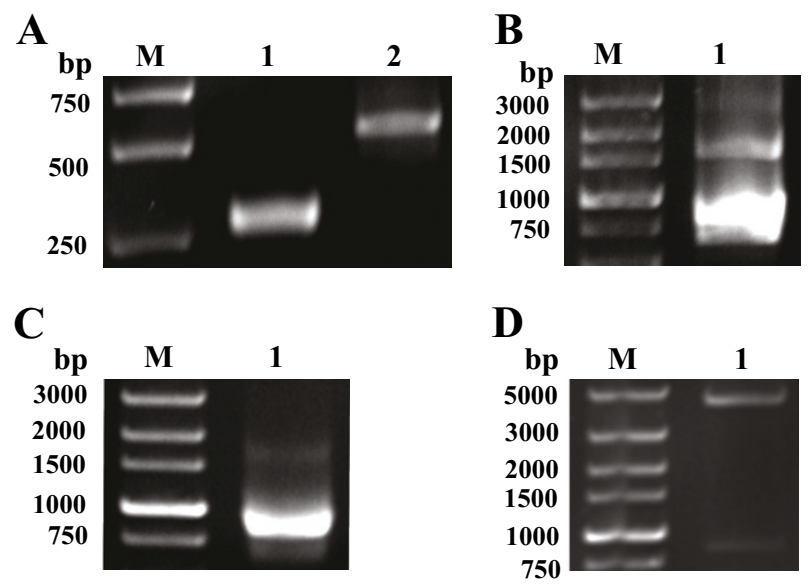

Figure 1. Cloning the ectodomain of $M a m u-B * 1703$ heavy chain and BSP fusion protein sequence into $\mathrm{pET}$-3d expression vector. A three-round PCR was performed for amplification of the fusion protein sequence. The products of each PCR round are respectively shown in (A), (B) and (C). Their expected sizes are $314 \mathrm{bp}$ and 569 bp (A, Lane 1 and Lane 2); 861 bp (B) and 895 bp (C). Finally, the expression vector was identified by $N c o$ I and BamH I cleavage (D) and direct DNA sequencing. M, DNA marker DL5,000.

I restriction site in the ectodomain sequence of Матu$B^{*} 1703$ (GenBank accession No. EU682520) (10), a pair of point mutation primers (Forword-mutBamH and BackmutBamH) was designed, and two fragments with the synonymous mutation were amplified respectively. Showing the expected lengths ( $314 \mathrm{bp}$ and $569 \mathrm{bp}$ ) (Figure 1A, Lanes 1 and 2), they were mixed together as the second-round template. The product of second-round PCR was $861 \mathrm{bp}$ in length (Figure 1B, Lane 1). The third-round PCR product with the expected length (895 bp) (Figure 1C, Lane 1) was digested with Nco I plus BamH I and inserted into pET-3d. The clone was identified by double enzyme cleavage to have the correct insert (Figure 1D, Lane 1) and was further confirmed by DNA sequencing, indicating that the recombinant plasmid (designated as pET-3d/Maти- $B^{*} 1703$ BSP) was constructed correctly.

Expression of Mamu-B*1703-BSP fusion protein in E. coli The plasmid $\mathrm{pET}-3 \mathrm{~d} /$ Mamu- $B^{*} 1703$-BSP was introduced into E. coli strain BL21 (DE3). After 4 hours of IPTG induction, a marked enhancement of a $33 \mathrm{kDa}$ protein expression was observed by SDS-PAGE analysis (Figure 2A, Lane 2). It accounted for about $20 \%$ of the bacterial total proteins, but mainly existed as inclusion body because it was hardly seen in the supernatant of the bacterial lysate (Figure 2A, Lane 3). Its molecular weight met well with that of the expected Mamu-B*1703-BSP fusion protein, and Western blotting analysis with anti-HLA-A*0201/NLV anti-serum further confirmed that the potential protein was the product of transformed $\mathrm{pET}-3 \mathrm{~d} /$ Maтu- ${ }^{*}$ 1703-BSP vector (Figure 2B).

Refolding and biotinylation of monomeric complex

Purified Mamu-B*1703-BSP inclusion body was refolded 

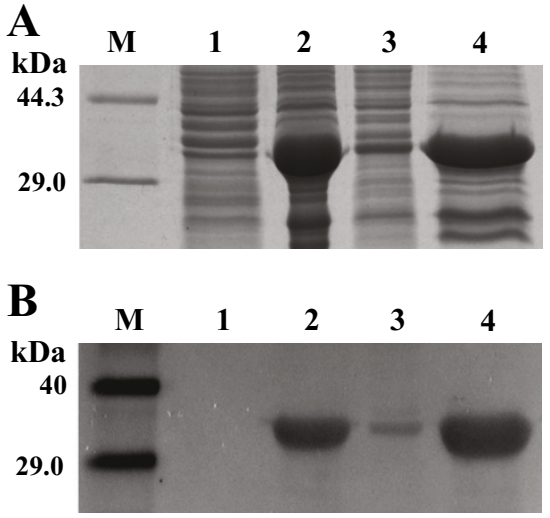

Figure 2. SDS-PAGE (A) and Western Blotting (B) analysis of the lysates of $E$. coli BL21 (DE3) transformed with a pET-3d control plasmid or with a Mamu-B*1703-BSP expression vector (Lanes 2-4). Lane 1, control plasmid; Lane 2, total lysate; Lane 3, supernatant; Lane 4, precipitate. M, protein markers.

with $\beta_{2} \mathrm{~m}$ and IW9 peptide in the refolding buffer (refolding mixture). Then the refolded Mamu-B*1703/IW9 monomer was biotinylated by BirA and purified by anion exchange resin in a MonoQ5/50 GL column. Three elution peaks were observed by monitoring of absorbance at $280 \mathrm{~nm}$. SDS-PAGE analysis showed that peak I was a $12 \mathrm{kDa}$ protein corresponding to unbound $\beta_{2} \mathrm{~m}$ light chain (Figure 3A, Lane
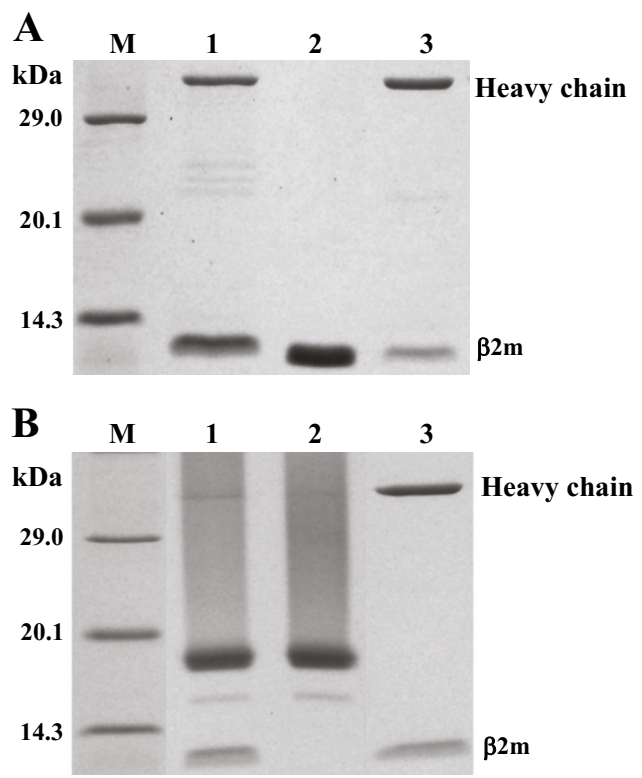

Figure 3. SDS-PAGE analysis of Mamu-B*1703/IW9 monomers (A) and tetramers (B). (A) Lane 1, refolded Mamu-B*1703/IW9 mixture before purification; Lane 2, peak I $\left(\beta_{2} \mathrm{~m}\right)$; Lane 3 , peak II (Mamu-B*1703/IW9 monomer). (B) Lane 1, Mamu-B*1703/IW9 monomers and PE-streptavidin mixture (noting that Mamu-B* 1703/IW9 tetramers were formed in the mixture); Lane 2, PEstreptavidin solution; Lane 3, biotinylated and purified Mamu-B* 1703/IW9 monomers. M, protein markers.

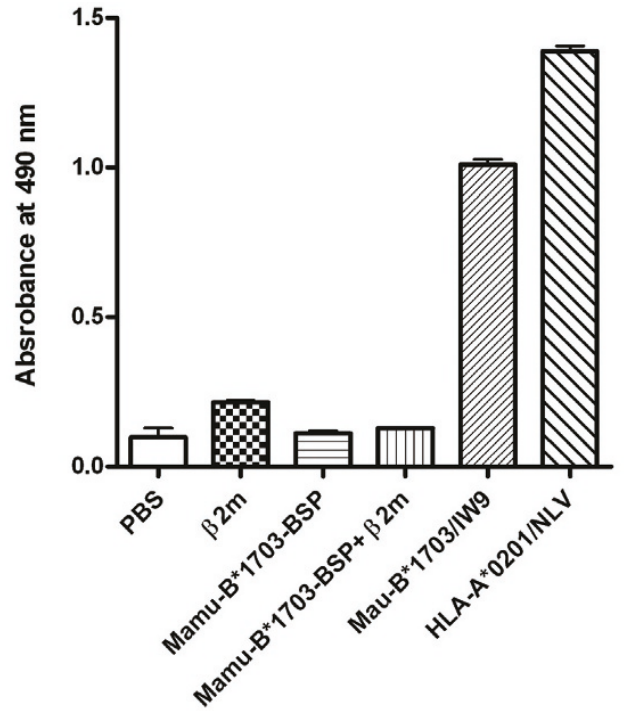

Figure 4. Identification of Mamu-B*1703/IW9 monomers' conformation by mAb W6/32 recognition. As shown in the figure, mAb W6/32 can hardly recognize free Mamu-B*1703-BSP fusion protein (heavy chain), $\beta_{2} \mathrm{~m}$ (light chain) or their mixture in absence of IW9 peptide. While Mamu-B*1703/IW9 monomer binds tightly with the W6/32. HLA-A*0201/NLV monomer and PBS are recruited as positive and negative controls respectively.

2) and peak II included a heavy chain $(33 \mathrm{kDa})$ and light chain (12 kDa) (Figure 3A, Lane 3), which indicated that they had been incorporated into a monomeric complex. Finally the biotinylated Mamu-B*1703/IW9 monomer was concentrated into $1 \mathrm{mg} / \mathrm{ml}$ by ultrafiltration.

\section{Preparation of Mamu-B*1703-IW9 tetramer}

Biotinylated Mamu-B*1703/IW9 monomers (Figure 3B, Lane 3) were mixed with one fourth of PE-streptavidin molecules (Figure 3B, Lane 2) to form tetramers. Under non-reducing condition and without being boiled, less than $10 \%$ of Mamu-B*1703/IW9 monomers remained free in the mixture (Figure 3B, Lane 1) by SDS-PAGE analysis, suggesting that more than $90 \%$ of the monomers were successfully biotinylated and polymerized.

\section{Conformation of Mamu-B*1703/IW9}

As mAb W6/32 recognizes an conformational determinant composed of both heavy chain and $\beta_{2} \mathrm{~m}$, it has been used to check whether the refolded MHC class I monomers have the correct conformation (16). The binding activities of Mamu-B*1703/IW9 monomer with an anti-MHC class I mAb W6/32 were determined by an ELISA assay. As shown in Figure 4, the absorbance values of soluble Mamu-B* 1703/IW9 and soluble HLA-A*0201/NLV (7) monomer wells were significantly higher than those of unfolded Mamu-B*1703-BSP, $\beta_{2} \mathrm{~m}$, Mamu-B*1703-BSP $+\beta_{2} \mathrm{~m}$, and PBS wells $(p<0.001)$. Mamu-B*1703/IW9 monomers could bind with mAb W6/32 as effectively as HLA-A*0201/NLV monomers did, while the same antibody could weakly 
recognize Mamu-B*1703-BSP fusion protein that was refolded in the presence or absence of $\beta_{2} \mathrm{~m}$ light chain. This result suggested that Mamu-B*1703/IW9 had the correct conformation as native MHC I molecules.

\section{Discussion}

In recent years, the Chinese-descent rhesus macaques have become more popular in HIV vaccine research than Indian macaques owing to their increased availability. But the critical reagents for vaccine research, such as MHC class I tetramers, are currently only designed on the immunogenetic background of Indian rhesus macaques, while the two geographic macaque populations share few common MHC class I alleles $(2,10,17-19)$. This study was intended to set up a procedure for constructing new tetramers on the immunogenetic background of Chinese macaques. In general, MHC class I alleles of Chinese-descent rhesus macaques were reverse-transcribed from the total RNA of the peripheral blood mononuclear cells, and cloned into pMD19-T vectors (10). The DNA fragment encoding the extracellular domain of MHC class I fused with an additional BirA substrate peptide (BSP) sequence was amplified from the identified vectors, and inserted into $\mathrm{pET}-3 \mathrm{~d}$ expression vectors. A monomer was constructed by refolding the expressed inclusion body in the presence of human $\beta_{2} \mathrm{~m}$ light chain and antigenic peptide. After biotinylation, the monomers were polymerized by PE-conjugated streptavidin. Our results proved successful preparation of MHC class I tetramers suitable for the Chinese-origin rhesus macaques, which suggests that the tetramers of other MHC class I alleles of Chinese macaques could be formulated using our procedure and therefore should facilitate the application of these available animals as vaccine models in general.

In the present study, we used human $\beta_{2} \mathrm{~m}$ in place of monkey $\beta_{2} \mathrm{~m}$. Our results showed that the Mamu-B*1703 heavy chain could be refolded with IW9 in the presence of human $\beta_{2} \mathrm{~m}$. This is consistent with the previous publication (20), which also reported that the monkey MHC I heavy chain could refolded with human $\beta_{2} \mathrm{~m}$ in construction of tetramers. The reason may be due to the high homology between human and monkey $\beta_{2} \mathrm{~m}$ (92\% identity). A structural analysis of human $\beta_{2} \mathrm{~m}$ and $M$. mulatta $\beta_{2} \mathrm{~m}$ suggests that the differences are insufficient to influence them to bind interchangeably with MHC I heavy chain of the other species (21). Together, these data suggest that human $\beta_{2} \mathrm{~m}$ can be used to prepare soluble monkey MHC I molecules in the presence of appropriate peptides.

Although MHC class I tetramers are the critical reagent for vaccine research and currently the "golden standard" for detecting specific $\mathrm{CD}^{+}$CTLs, it will be impossible to prepare a tetramer without knowing the appropriate antigenic epitopes. However, it is a laborious and time-consuming work to identify appropriate antigenic peptides for a specific MHC class I molecule. Due to the shortage of appropriate data of antigenic epitopes of Mamu-B*1703 molecules, antigenic peptides reported to be restricted by MHC class I alleles of Indian macaques may be taken into account in preparation of Chinese-descent macaque MHC class I tetramers. Although Chinese- and Indian-descent macaques share few identical MHC class I alleles, some reported antigenic peptides may be presented commonly by highly homologous MHC class I molecules of the two macaque populations. This phenomenon has been recognized in human MHC class I alleles. Many epitope peptides containing certain features can bind with several alleles and the features are used to define a "supermotif", while these alleles are called "supertype". In the present study the sequence of Mamu- $B^{*} 1703$, which was identified by our group (10) as a Chinese-descent macaque MHC class I allele, is similar to that of Mamu- $B^{*} 1701$ of Indian macaques. And it was our hypothesis that $M a m u-B^{*} 1701$-restricted epitope of SIVnef protein, IW9 peptide, could also be recruited as a ligand in construction of Mamu-B*1703 tetramer. Another reason why we selected this epitope was that SIVnef protein is one of the early expressed viral accessory proteins, and it is often a major object of SIV vaccines to elicit CTL responses to this protein or a combination of this protein with other early and late expressed ones (Tat and Gag, etc.) $(1,22-24)$. In the presence of IW9 peptide, Mamu-B*1703 heavy chain and $\beta_{2} \mathrm{~m}$ light chain were effectively bound together as a monomer. While without the presence of IW9 peptide, no significant soluble Mamu-B*1703 monomer was formed (data not shown). This result indicated that IW9 could bind firmly with Mamu-B*1703 and facilitate the correct refolding of the monomers. Thus, IW9 may contain some feature of a supermotif that could be recognized by a potential Maтu- $B^{*} 17-$ like supertype represented by Mamu- $B^{*} 1701$ and $B^{*} 1703$, yet extensive investigation are needed to verify this hypothesis.

As mentioned above, soluble Mamu-B*1703/IW9 monomers were obtained in the presence of the IW9 peptide, which means that this antigenic peptide may be presented on the surface by $M a m u-B * 1703$ complexes whereby induces specific $\mathrm{CD} 8^{+} \mathrm{CTL}$ responses. But the binding activity to cognate monkey $\mathrm{CD} 8^{+}$CTLs remains to be verified using SIV-infected Maти- $B^{*} 1703$-positive macaques. Due to the shortage of this resource, its conformation was confirmed by $\mathrm{mAb}$ W6/32 recognition instead of SIV-activated CD8 ${ }^{+}$CTL staining. Previous reports have shown that the anti-MHC class I mAb W6/32 can tightly bind with MHC class I complex of correct conformation, and weakly do the free heavy chain while hardly recognize the free $\beta_{2} \mathrm{~m}$ light chain (16). Although $\mathrm{W} 6 / 32$ is originally developed for human class I MHC molecules and it only recognizes properly refolded MHC I heavy chain binding with light chain (16, 25 ), it is suggested that W6/32 should react similarly with human or macaque MHC class I antigens that are bound with $\beta_{2} \mathrm{~m}$ of either species (human-macaque chimeric monomers) (21). The fact that $\mathrm{mAb} W 6 / 32$ could recognize Mamu-B*1703/IW9 monomer as it do HLA-A*0201 monomer loaded with a HCMV peptide, which has been successfully applied in investigation of HCMV-specific CD8 ${ }^{+}$ $\mathrm{T}$ cells in Chinese populations (7-9), implicates that Mamu-B*1703/IW9 has been properly refolded in the new 
tetramer. To the best of our knowledge, Mamu-B*1703/IW9 tetramer constructed by our group was the first tetramer based on the MHC class I background of Chinese macaques. It is a potential tool for investigation of the frequency, phenotype and function of SIV-specific $\mathrm{CD}^{+}$CTLs in vaccine models of Chinese-macaques.

\section{Acknowledgements}

This work was supported by Natural Science Fund of Guangdong Province (No.8451063201000340) and the Talented Man Initiation Fund of Jinan University (No.51208004, No.51208017) to Dr. DY Ouyang and grants from the National Natural Science Foundation of China (No.30572199, No.30230350 and No.30371651) to Prof. XH $\mathrm{He}$; as well as by the Biochemistry and Molecular Biology Key Discipline of Guangdong Province.

\section{References}

1. Feinberg MB, Moore JP. AIDS vaccine models: challenging challenge viruses. Nat Med. 2002;8:207-210.

2. Karl JA, Wiseman RW, Campbell KJ, et al. Identification of MHC class I sequences in Chinese-origin rhesus macaques. Immunogenetics. 2008;60:37-46.

3. Ling B, Veazey RS, Luckay A, et al. SIV(mac) pathogenesis in rhesus macaques of Chinese and Indian origin compared with primary HIV infections in humans. AIDS. 2002;16:1489-1496.

4. Reimann KA, Parker RA, Seaman MS, et al. Pathogenicity of simian-human immunodeficiency virus SHIV-89.6P and SIVmac is attenuated in cynomolgus macaques and associated with early T-lymphocyte responses. J Virol. 2005;79:8878-8885.

5. Marthas ML, Lu D, Penedo MC, Hendrickx AG, Miller CJ. Titration of an SIVmac251 stock by vaginal inoculation of Indian and Chinese origin rhesus macaques: transmission efficiency, viral loads, and antibody responses. AIDS Res Hum Retroviruses. 2001;17:1455-1466.

6. Sun Y, Iglesias E, Samri A, et al. A systematic comparison of methods to measure HIV-1 specific CD8 T cells. J Immunol Methods. 2003;272:23-34.

7. He XH, Xu LH, Liu Y. Procedure for preparing peptide-major histocompatibility complex tetramers for direct quantification of antigen-specific cytotoxic T lymphocytes. World J Gastroenterol. 2005;11:4180-4187.

8. He XH, Zha QB, Liu Y, Xu LH, Chi XY. High frequencies cytomegalovirus pp65(495-503)-specific $\mathrm{CD}^{+} \mathrm{T}$ cells in healthy young and elderly Chinese donors: characterization of their phenotypes and TCR V $\beta$ usage. J Clin Immunol. 2006;26: 417-429.

9. He XH, Jia QT, Li FY, et al. $\mathrm{CD}^{+} \mathrm{T}$ cells specific for both persistent and non-persistent viruses display distinct differentiation phenotypes but have similar level of PD-1 expression in healthy Chinese individuals. Clin Immunol. 2008; 126:222-234.

10. Ouyang D, Xu L, Dai Z, et al. Identification of major histocompatibility complex class I alleles in Chinese rhesus macaques. Acta Biochim Biophys Sin (Shanghai). 2008;40:919-
927.

11. Minang JT, Trivett MT, Coren LV, et al. The Mamu B 17-restricted SIV Nef IW9 to TW9 mutation abrogates correct epitope processing and presentation without loss of replicative fitness. Virology. 2008;375:307-314.

12. Mothe BR, Sidney J, Dzuris JL, et al. Characterization of the peptide-binding specificity of Mamu-B*17 and identification of Mamu-B*17-restricted epitopes derived from simian immunodeficiency virus proteins. J Immunol. 2002;169:210- 219.

13. Jia Q, Xu L, Zha Q, Chi X, Li F, He X. High level expression of HLA-A*0203-BSP fusion protein in Escherichia coli and construction of soluble HLA-A*0203 monomer and tetramer loaded with Epstein-Barr virus peptide. Cell Mol Immunol. 2007;4:301-308.

14. He XH, Xu LH, Liu Y, Zeng YY. Cloning of human $\beta$-microglobulin gene and its high expression in Escherichia coli. Sheng Wu Gong Cheng Xue Bao. 2004;20:99-103.

15. Laemmli UK. Cleavage of structural proteins during the assembly of the head of bacteriophage T4. Nature. 1970;227: 680-685.

16. Lu X, Wu X, Liang Z, Weng X, Li Q, Gong F. Construction and functional test of HLA-A*2402-peptide tetramer. Cell Mol Immunol. 2005;2:145-149.

17. Otting N, Heijmans CM, van der WM, de Groot NG, Doxiadis GG, Bontrop RE. A snapshot of the Mamu-B genes and their allelic repertoire in rhesus macaques of Chinese origin. Immunogenetics. 2008;60:507-514.

18. Otting N, Heijmans CM, Noort RC, et al. Unparalleled complexity of the MHC class I region in rhesus macaques. Proc Natl Acad Sci U S A. 2005;102:1626-1631.

19. Otting N, de Vos-Rouweler AJ, Heijmans CM, de Groot NG, Doxiadis GG, Bontrop RE. MHC class I A region diversity and polymorphism in macaque species. Immunogenetics. 2007;59: 367-375.

20. Kuroda MJ, Schmitz JE, Barouch DH, et al. Analysis of Gagspecific cytotoxic $\mathrm{T}$ lymphocytes in simian immunodeficiency virus-infected rhesus monkeys by cell staining with a tetrameric major histocompatibility complex class I-peptide complex. J Exp Med. 1998;187:1373-1381.

21. Su J, Luscher MA, MacDonald KS. Sequence of $\beta_{2^{-}}$ microglobulin from rhesus macaque (Macaca mulatta) includes an allelic variation in the 3 '-untranslated region. Immunogenetics. 2004;55:873-877.

22. Evans TG, Keefer MC, Weinhold KJ, et al. A canarypox vaccine expressing multiple human immunodeficiency virus type 1 genes given alone or with rgp120 elicits broad and durable $\mathrm{CD}^{+}$cytotoxic $\mathrm{T}$ lymphocyte responses in seronegative volunteers. J Infect Dis. 1999;180:290-298.

23. Sharpe S, Polyanskaya N, Dennis M, et al. Induction of simian immunodeficiency virus (SIV)-specific CTL in rhesus macaques by vaccination with modified vaccinia virus Ankara expressing SIV transgenes: influence of pre-existing anti-vector immunity. J Gen Virol. 2001;82:2215-2223.

24. Gallimore A, Cranage M, Cook N, et al. Early suppression of SIV replication by $\mathrm{CD}^{+}$nef-specific cytotoxic $\mathrm{T}$ cells in vaccinated macaques. Nat Med. 1995;1:1167-1173.

25. Garboczi DN, Hung DT, Wiley DC. HLA-A2-peptide complexes: refolding and crystallization of molecules expressed in Escherichia coli and complexed with single antigenic peptides. Proc Natl Acad Sci U S A. 1992;89:3429-3433. 